\title{
Revisión histórica y conceptual de la autoría y sus implicaciones en el medio televisivo: el concepto de autor en las series de televisión contemporáneas estadounidenses ${ }^{12}$
}

\section{Historical and conceptual review of authorship and its implications for television media: the concept of author in American contemporary fiction TV-series}

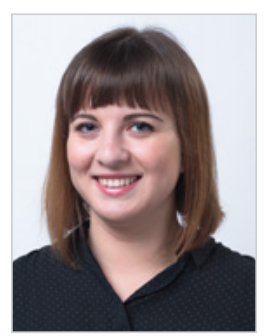

\begin{abstract}
María-José Higueras-Ruiz. Estudiante de doctorado de Ciencias Sociales en el Departamento de Información y Comunicación de la Universidad de Granada, y contratada predoctoral FPU (Formación de Profesorado Universitario) del Ministerio de Educación, Cultura y Deporte del Gobierno de España. Es miembro del Grupo de Investigación “CommuniCAV” (PAI SEJ-585), y del Proyecto de Investigación de Excelencia “Transmedialización y crowdsourcing en las narrativas de ficción y no ficción audiovisuales, periodísticas, dramáticas y literarias” (Ref CSO2017-85965-P). Ha asistido y participado en congresos nacionales e internacionales sobre comunicación, y ha publicado varios artículos en destacadas revistas científicas. Su actividad investigadora versa sobre la producción de series de ficción televisiva, y el perfil del showrunner como productor ejecutivo-creativo de dichos proyectos audiovisuales.

Universidad de Granada, España

mhiguer@ugr.es
\end{abstract}

ORCID: 0000-0002-6849-3433

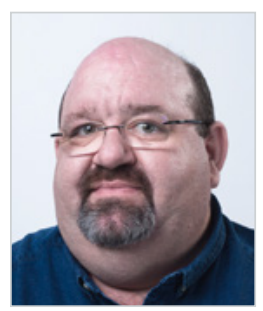

Francisco-Javier Gómez-Pérez. Profesor Contratado Doctor en el Departamento de Información y Comunicación de la Universidad de Granada. Es miembro del Grupo de Investigación "CommuniCAV” (PAI SEJ-585), y del Proyecto de Investigación de Excelencia “Transmedialización y crowdsourcing en las narrativas de ficción y no ficción audiovisuales, periodísticas, dramáticas y literarias" (Ref. CSO2017-85965-P). Es autor del libro Consolidación industrial del cine andaluz (2013), y editor del volumen Políticas de impulso a las industrias audiovisuales (2015). Asimismo, ha publicado numerosos trabajos científicos y ha coordinado congresos, reuniones de investigación, seminarios y foros educativos y empresariales, sobre el proceso de producción audiovisual.

Universidad de Granada, España

frangomez@ugr.es

ORCID: 0000-0001-7539-1681

1 El presente artículo ha sido elaborado durante la contratación pre-doctoral: "Formación de Profesorado Universitario" (FPU 15/00737) del Ministerio de Educación, Cultura y Deporte del Gobierno de España.

2 Proyecto de Investigación de I+D de Plan Nacional «Transmedialización y crowdsourcing en las narrativas de ficción y no ficción audiovisuales periodísticas, dramáticas y literarias», (2018-2020) (Ref. CSO2017-85965-P). Domingo Sánchez-Mesa Martínez y Jordi Alberich-Pascual (2018-2020).

Cómo citar este artículo:

Higueras-Ruiz, M. J.; Gómez-Pérez, F. J.y Alberich-Pascual, J. (2019). Revisión histórica y conceptual de la autoría y sus implicaciones en el medio televisivo: el concepto de autor en las series de televisión contemporáneas estadounidenses. Doxa Comunicación, 28, pp. 79-96.

https://doi.org/10.31921/doxacom.n28a04 


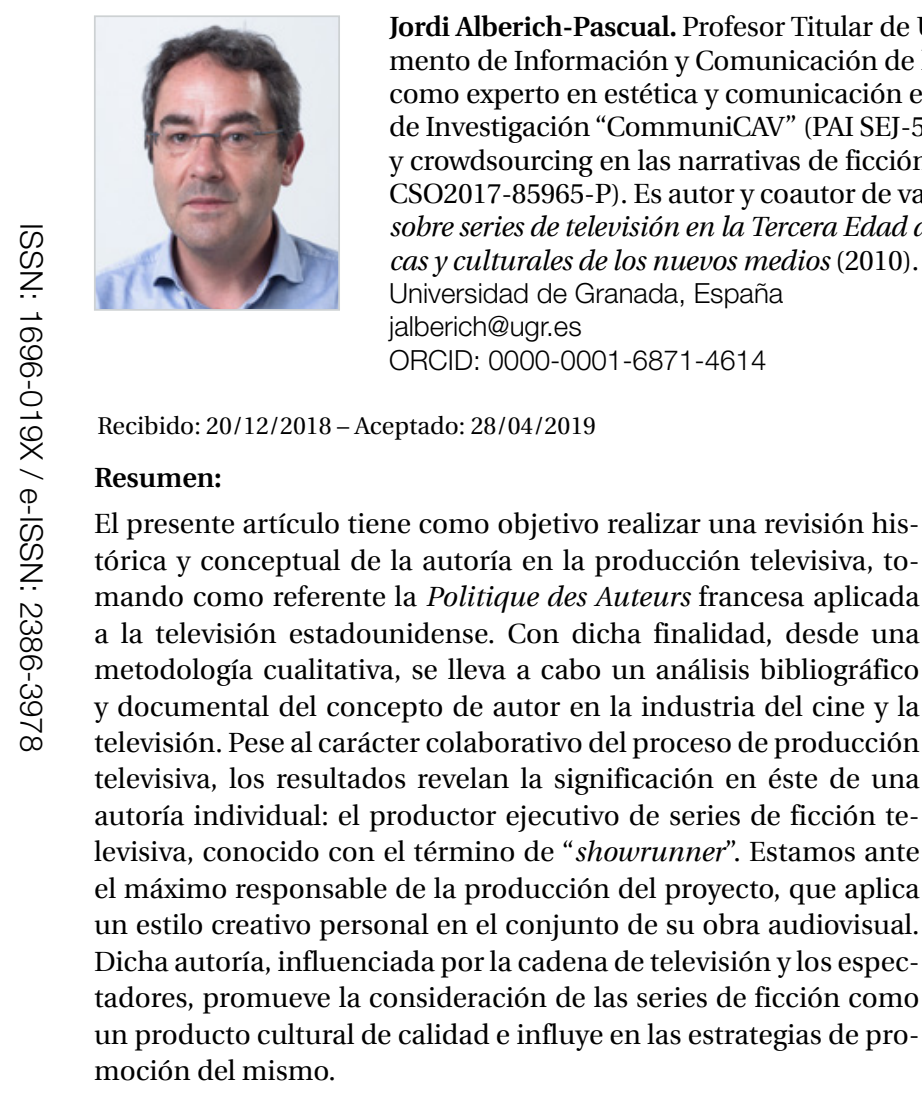

\section{Palabras clave:}

Autoría; showrunner, series de televisión; teoría del autor; proceso colaborativo.

\section{Received: 20/12/2018 - Accepted: 28/04/2019}

\section{Abstract:}

This paper aims to conduct a historical and conceptual review of authorship in television production, taking auteur theory applied to the television media as a reference. For this purpose, the qualitative methodology has been used to carry out a bibliographic and documentary analysis of the author concept in the film and television industry. Despite the collaborative nature of television production, the results obtained suggest the importance of the TV series executive producer's individual authorship during this process, who is also known as the "showrunner". They are ultimately responsible for the project's production, applying a creative and personal style to their entire work. The authorship, influenced by the television channel and the audience, promotes the consideration of fiction TV series as a quality cultural product, which affects its promotional strategies.

\section{Keywords:}

Authorship; showrunner; TV series; auteur theory; collaborative process. 


\section{Introducción}

La asignación de la autoría de un proyecto creativo constituye una tarea compleja, propiciando que críticos y académicos pertenecientes a diferentes medios -literatura, cine y televisión- muestren su interés hacia dicha cuestión. En este sentido, numerosos investigadores han revisado las aportaciones en torno al concepto de autor en el cine y la literatura, cuyos resultados constituirán la base para el estudio en el medio televisivo (Mittell, 2015).

La Politique des Auteurs ha experimentado diversas articulaciones, así como críticas y modificaciones desde su origen. De forma genérica, consiste en defender la posición del director como autor de la película, y su actuación, como expresión creativa y personal, lo que configura una huella reiterativa en el conjunto de su obra fílmica (Cuevas, 1994; Sanderson, 2005; Pellejero, 2012; Chaudhuri, 2013; Galindo-Pérez, 2015).

En la era contemporánea resulta esencial hacer referencia a este paradigma en la industria televisiva, señalando el aumento y la popularidad de la producción de series de ficción, lo que incrementa la atención de críticos y periodistas hacia dicho medio (Schatz, 2014; Perren y Schatz, 2015).

Así, diversos textos asignan la autoría en televisión al productor ejecutivo-creativo y valoran la consideración del showrunner as auteur (Newman y Levine, 2012; Blakey, 2017). Siguiendo esta idea, Kompare (2011) incide en tres nociones relacionadas: la producción televisiva es entendida como un proceso colaborativo, la autoría en televisión supone una marca que permite a las cadenas competir en el mercado gracias a la firma autoral de los creadores de las series de ficción que emiten, y este fenómeno contribuye a la formación de un espectador culto y al incremento del interés suscitado por los círculos académicos. Para comprender la noción de autor en el medio televisivo debemos considerar su actuación dentro de un contexto económico y cultural determinado (Mittell, 2015; Blakey, 2017), advirtiendo el complejo sistema de comunicación que conlleva dicho proceso colaborativo, lo que dificulta la tarea de discernir quién es el autor de la obra (Cantor, 1988; Thompson y Burns, 1990; Kubey, 2009; Mann, 2009). A pesar de ello, Hills (2013: 201) expone que "lejos de dejar de ser un tema de interés académico y para la audiencia, la autoría se ha convertido en un factor cada vez más importante para la industria de la televisión, con la adopción de un modelo de 'showrunner' al estilo de los Estados Unidos”3.

Podemos destacar trabajos previos, como el de Newcomb y Alley (1983) - The Producer's Medium-, que ya asignaban el control creativo en televisión al productor ejecutivo, cuya visión personal y habilidad para articular las aportaciones de los diferentes profesionales eran determinantes para el resultado final del proyecto. No obstante, en el siglo XXI las citadas cuestiones se ven acentuadas gracias a la popularidad adquirida por el showrunner que, según Newman y Levine (2012: 38), "es potencialmente un autor, un artista con una visión única cuyas experiencias y personalidad se expresan a través del oficio de contar historias, y cuya presencia en los discursos culturales funciona para producir autoridad en las formas con las que se identifica"4.

3 Texto original: "far from receding as a topic of academic and audience interest, authorship has become an increasingly important factor for the TV industry, with a US-style 'showrunner' model being adopted” (Hills, 2013: 201). Traducción propia.

4 Texto original: "is potentially an auteur, an artist of unique vision whose experiences and personality are expressed though storytelling craft, and whose presence in cultural discourses functions to produce authority for the forms with which he is identified” (Newman and Levine, 2012: 38). Traducción propia. 
Revisión histórica y conceptual de la autoría y sus implicaciones en el medio televisivo...

\section{Objetivos y metodología}

El objetivo principal del presente trabajo consiste en revisar y analizar la autoría en el medio televisivo estadounidense, aplicando a dicha industria las ideas derivadas de la Politique des Auteurs. Para ello, desde una metodología cualitativa, se revisa la bibliografía especializada en ambos contextos: desde los artículos publicados en la revista francesa de cine Cahiers du Cinema, hasta, especialmente, los textos que evalúan la atribución de la autoría televisiva al perfil del showrunner en la era contemporánea.

Con dicha finalidad, el artículo se estructura en tres partes que desarrollan la investigación propuesta. En primer lugar, como premisa del estudio, consideramos la producción audiovisual como un proceso colaborativo. Para ello, acudimos a las aportaciones de Scott (1975), Sanderson (2005) y Chaudhuri (2013). De forma específica en el contexto televisivo, resulta de especial interés el trabajo de Thompson y Burns (1990), y las investigaciones realizadas por Cantor y Cantor (1992), Kubey (2009), Mann (2009), Kompare (2011) y Blakey (2017).

A continuación, se revisará la extensa bibliografía acerca de la Politique des Auteurs en el contexto francés, a través de los volúmenes que recopilan artículos y entrevistas publicadas en Cahiers du Cinéma (Santos-Fontenla, 1974; Baecque, 2003; Romaguera y Alsina, 2010). Asimismo, analizamos las aportaciones de Sarris (1962, 1967, 1968) y Wollen (1969), que desarrollan dicho paradigma en Estados Unidos, donde se denominó Auteur Theory-empleamos el término "politique" o "theory" en función del contexto-.

Finalmente, con el objetivo de estudiar la autoría en la industria televisiva, consideramos los trabajos de Dunne (2007), Lotz (2009), Hadas (2014), Schatz (2014), Steiner (2015), Benshoff (2016), y Fisk y Szalay (2017). En este punto, se revela imprescindible el libro de Gray y Johnson (2013): Companion to Media Authorship. Del mismo modo, resultan especialmente pertinentes los textos de Newman y Levine (2012) y de Mittell (2015).

Esta revisión bibliográfica nos ha permitido sintetizar las conexiones entre la Politique des Auteurs y la consideración del showrunner como autor de las series de ficción televisiva en el siguiente gráfico. El contenido del mismo ha guiado y facilitado el posterior análisis crítico de cada uno de los conceptos. 
Figura 1: Conexiones entre la autoría en el cine y la televisión.

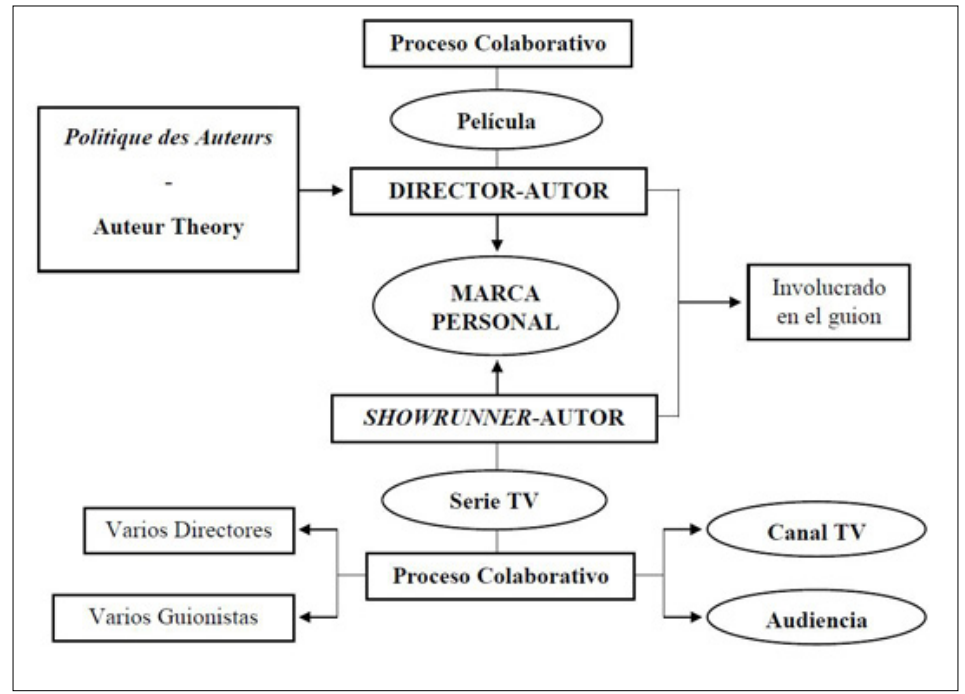

Fuente: Recopilación basada en la información proporcionada por la revisión bibliográfica.

Los resultados obtenidos se presentan estructurados en tres bloques de contenido. Los dos primeros, de carácter preliminar, advierten la premisa de considerar la producción televisiva como un proceso colaborativo, y exponen las principales aportaciones a la autoría cinematográfica realizadas por los críticos franceses y los teóricos norteamericanos. La tercera parte, foco central de este artículo, analiza y estudia la aplicación de la teoría del autor en el medio televisivo, destacando las dinámicas de autoría asignadas al productor ejecutivo como máximo responsable de la serie de ficción.

\section{Resultados}

\subsection{La producción televisiva como proceso colaborativo}

La producción audiovisual supone un proceso colaborativo donde intervienen diferentes profesionales, junto con la maquinaria y el respaldo económico. Estamos ante una creatividad colectiva que implica la interacción de los miembros de un equipo para realizar una sola obra (Scott, 1975). Siguiendo esta línea, Santos-Fontenla (1974: 11) incide en que "durante mucho tiempo se ha negado al cineasta la posibilidad de ser considerado 'autor' de sus obras en virtud de tratarse el cine de un trabajo en equipo". Del mismo modo, Sanderson (2005) anota que, a pesar de la responsabilidad asignada al director, la participación de un grupo de personas impide asignar la creación de la película a un único profesional.

Si consideramos el medio televisivo de forma específica, la implicación de diferentes personas es aún más acentuada en la producción de series de ficción, lo que dificulta la tarea de determinar su autoría (Newcomb y Alley, 1983; Mittell, 2015; Jen- 
sen, 2017). Campbell y Reeves (1990: 8) expresan que "es un error pensar en la autoría televisiva únicamente en términos de la expresión de la visión artística de una persona"5. Del mismo modo, Benshoff (2016) advierte la complejidad de reconocer al autor de dichos proyectos audiovisuales, que son escritos por un grupo de guionistas y dirigidos por varios directores, sin obviar la intervención de los directivos y la imagen de la cadena.

Sin embargo, a pesar de la crítica a la autoría individual, dicha perspectiva no impide la asignación de una voz autoral perteneciente a una persona o a un grupo creativo, donde se incluye al productor ejecutivo (Mann, 2009; Tous-Rovirosa, 2009; Blakey, 2017). En el contexto cinematográfico, puede "darse el caso de una película donde el productor tenga derecho a ser considerado tan autor de la obra resultante como el director o el guionista. Ahora bien, esto solo se entiende desde la consideración del cine como un trabajo creativo conjunto, un arte colaborativo" (Pardo, 2000: 247). En cuanto a la industria televisiva, Guerrero (2013) expresa que "la producción audiovisual se considera, fundamentalmente, un trabajo en equipo cuyo liderazgo es asumido por el productor ejecutivo" (48); y Newman y Levine (2012: 38) añaden: “Las series de televisión [...] son escritas por las personas que las crean, tanto si se entiende que estos autores funcionan como individuos autónomos o como un equipo de colaboradores que trabajan juntos" ${ }^{\text {. }}$

Por lo tanto, la autoría en televisión se entiende y analiza dentro de un contexto colaborativo donde "el autor debe coordinar y facilitar los esfuerzos conjuntos de un equipo grande y complejo"7 (Campbell y Reeves, 1990: 14). El productor ejecutivo es la persona designada para dicha labor, al ejercer la máxima responsabilidad sobre el proyecto y tomar decisiones en cada fase de su producción (Thompson y Burns, 1990). De este modo, "el producto terminado de un episodio transmitido pasa por un complejo proceso de colaboración, filtrando las contribuciones de los intérpretes, diseñadores, editores y ejecutivos de la cadena, pero la responsabilidad del producto final recae sobre el showrunner"8 (Mittell, 2015: 91).

Este profesional debe establecer una línea narrativa, estética y temática -conocida como la "voz del showrunner" (Bennett, 2014; Kirkpatrick, 2015)-, y asegurar su consistencia durante cada episodio de la serie (Newcomb y Alley, 1983; Perren, 2011; Newman y Levine, 2012). El objetivo es evitar que las presiones ejercidas por los diferentes agentes implicados desvirtúen la visión original (Kubey, 2009).

En este punto se revela esencial incidir en el autor de la obra audiovisual desde una perspectiva legal, lo que resulta especialmente complejo debido a la ausencia de una normalización jurídica sobre la propiedad intelectual a escala internacional. Conviven dos sistemas legales distintos: el anglosajón -EE.UU. y Gran Bretaña, principalmente- y el latino -donde se incluye España-, lo que supone diferentes consideraciones sobre los derechos de autor y la propiedad intelectual (Pardo, 2009). En el primer caso, "el productor es el titular último de los derechos de autor sobre la obra audiovisual, modalidad sin duda más ventajosa para la industria y claramente más moderna” (Sáinz-Sánchez, 1999: 100). En el caso de la legislación española -Ley 23/2006, de 7 de julio, que modifica el texto refundido del Real Decreto 11/1996, de 12 de abril-, son consi-

5 Texto original: "it's a mistake to think of television authorship solely in terms of the expression of an individual's artistic vision" (Campbell and Reeves, 1990: 8). Traducción propia.

6 Texto original: "Television shows [...] are authored by the people who create them, whether these authors are understood to function as autonomous individuals or as a team of collaborators working together" (Newman and Levine, 2012: 38). Traducción propia.

7 Texto original: "the television author must coordinate and facilitate the concerted efforts of a large and complex team" (Campbell and Reeves, 1990: 14). Traducción propia.

8 Texto original: "the final product of an aired episode goes through complex collaborative process, filtering the contributions of performers, designers, editors, and network executives, but the responsibility for the end product rests with the showrunner" (Mittell, 2015: 91). Traducción propia. 
derados autores el director, el guionista ${ }^{9} \mathrm{y}$ el compositor, que poseen derechos morales. Por su parte, el productor ostenta derechos patrimoniales: de explotación y remuneratorios (Pardo, 2014). Dicha ley diferencia entre los conceptos de obra en colaboración-como define a la obra audiovisual-donde diferentes autores poseen los derechos en cotitularidad; y el de obra colectiva-concepto más próximo a la mentalidad legal anglosajona-, donde una única persona -el productor-coordina las contribuciones de varios autores y posee los derechos de propiedad intelectual (Pardo, 2009).

Por otra parte, numerosos estudios contemplan la posibilidad de asignar parte de la autoría a otros agentes relacionados: la cadena de televisión y el espectador. La cadena ejerce un constante control sobre la producción de la serie de ficción, tomando la decisión final acerca de su emisión (Cantor, 1988; Kubey, 2009). Por lo tanto, la existencia de una relación positiva entre los ejecutivos de la misma y el showrunner será esencial para la calidad del proyecto (Campbell y Reeves, 1990). Además, debemos advertir un conjunto de parámetros establecidos por las cadenas -tono, contenido, género- para determinar los proyectos que financian y emiten, con la finalidad de atraer a una tipología determinada de público (Hills, 2013). Dichas características le permiten configurar una identidad asociada al estilo autoral de las series de ficción que distribuyen, especialmente a través de la producción de contenido original (Johnson, 2013; Mittell, 2015).

Los canales tradicionales estadounidenses -ABC, NBC, y CBS- comienzan a modificar su modelo de producción en este sentido, pero se sitúa en los de cable -concretamente en $\mathrm{HBO}$ - el inicio de la búsqueda de la imagen de marca. Por lo tanto, “HBO se convierte en el 'autor' de sus propios programas junto con las firmas autorales canónicamente reconocidas. Ya no es solo importante que sea un programa de o creado por, es importante que se trate de una Programación Original de $H B O " 10$ (Maio, 2011: 279).

Asimismo, apreciamos mayor libertad creativa y autonomía autoral en los canales de cable en la era contemporánea (Tous-Rovirosa, 2009; Perren, 2011; Dunleavy, 2018). Siguiendo esta idea, Steiner expone:

Aunque [el canal de cable HBO] no inventó poner el foco de atención en el showrunner-auteur, la cadena lo ha convertido en una forma de arte en sí misma, para construir la imagen pública de un paraíso creativo de calidad prometedora, donde los guionistas cuentan con el máximo apoyo y la mínima interferencia ${ }^{11}$ (Steiner, 2015: 183).

A pesar de ello, "incluso si los showrunners tienen menos voces ante las que responder en los canales de cable, aún deben negociar con las entidades que financian, distribuyen y comercializan sus programas"12 (Perren, 2011: 138), ajustando sus contenidos a los parámetros demandados por cada cadena de televisión.

9 En el contexto que nos ocupa consideramos conveniente recordar el perfil de guionista que la figura del showrunner suele desarrollar.

10 Texto original: "HBO si fa 'autore' dei propri programmi affiancandosi alle firme autoriali canónicamente riconosciute. Non è più solo importante che sia un programma di o creato da; è importante che sia un HBO Original Programming" (Maio, 2011: 279). Traducción propia.

11 Texto original: "Although it [cable network HBO's] did not invent putting the focus of attention on one showrunner-auteur, the network has made it an art form in itself to build a public image of a creative haven promising quality, where writers are provided with a maximum of support and a minimum of interference" (Steiner, 2015: 183). Traducción propia.

12 Texto original: "even if showrunners may have fewer voices to answer to in cable, they still must negotiate with the entities that finance, distribute, and market their programs" (Perren, 2011: 138). Traducción propia 
En relación con la audiencia, el espectador se implica en el proyecto televisivo a través de las opciones transmedia, la interacción con el showrunner y la participación en la creación de contenidos, gracias a la tecnología de internet y a las redes sociales. Dichas oportunidades abren una nueva vía a la "co-creación" de series de ficción en la era digital (Gray y Johnson, 2013).

\subsection{La Politique des Auteurs}

La Politique des Auteurs fue promovida y estimulada dentro de un contexto cultural y social francés en el que surgieron varias revistas cinematográficas, cineclubes, filmotecas y festivales de cine (Stam, 2001). Dicho movimiento encuentra su origen en la Nouvelle Vague o French New Wave y, específicamente, en un grupo de cineastas y críticos franceses que formaban parte de la revista de cine Cahiers du Cinéma. El principal propulsor del paradigma fue François Truffaut, quien en 1954 publica Une certaine tendance du cinema français, donde acuña y expone las características de la política de los autores. La principal premisa se halla en la idea de que el director de cine, considerado el autor de la película, expresa una visión personal a través del empleo de un determinado estilo en la realización de la misma (Chaudhuri, 2013). Así, se valora la puesta en escena como herramienta de dicho profesional para imprimir su huella sobre el proyecto (Santos-Fontenla, 1974), de manera que el "cómo se dice" es un claro reflejo de la actuación y personalidad del autor (Casetti, 1994; Stam, 2001). Los artículos de Cahiers du Cinéma aprecian la estética de la película por encima de su temática, y "el autor llega a ser más importante que el propio filme. La firma certifica el valor de la obra y garantiza su calidad" (Casetti, 1994: 96). En este sentido, Truffaut comparte que "no hay buenas y malas películas, solo buenos y malos directores"13 (Chaudhuri, 2013: 80).

Dicho autor advierte que el estilo aplicado por los directores llega a ser un elemento reiterativo en el conjunto de su obra, proporcionándole continuidad y permitiendo que la audiencia y la crítica reconozcan estas huellas (Scott, 1975; Stam, 2001), que se configuran "como testimonios de una visión, de una personalidad individual" (Galindo-Pérez, 2015: 53). En este punto, para comprender la actuación del director, debemos destacar su relación con el guion de la película, que incluye la competencia para cambiar diálogos y escenas durante la fase del rodaje (Chaudhuri, 2013).

Posteriormente, Eric Rohmer publica el artículo Les lecteurs des "Cahiers" et la Politique des Auteurs (1956) para estudiar las anteriores cuestiones (Pellejero, 2012). Asimismo, expone la admiración hacia los cineastas americanos en Redécouvrir l'Amérique (1955), donde afirma: "Si tuviera que caracterizar el estilo del cine estadounidense, propondría dos palabras: eficacia y elegancia" ${ }^{14}$ (Rohmer, [1955] 1985: 89). Los críticos de Cahiers du Cinéma consideraban que los directores de Hollywood, a pesar de trabajar dentro del sistema de estudios, eran autores que aplicaban elementos estilísticos y temáticos personales en sus obras (Benshoff, 2016).

Otra de las aportaciones más destacables es la de Alexandre Astruc. En el artículo Du Stylo à la caméra et de la caméra au stylo ([1948] 1999) incide en la libertad de expresión permitida por el cine, y asemeja la labor del director a la del escritor, y la de la cámara de vídeo a la de la estilográfica. El autor inicia un debate sobre las competencias del guionista y del director, defendiendo que la situación ideal es aquella en la que ambos perfiles coinciden (Astruc, [1948] 1999). Esto supone "tanto

13 Texto original: "there are no good and bad movies, only good and bad directors" (Chaudhuri, 2013: 80). Traducción propia.

14 Texto original: "If I had to characterize the American style of cinema, I would put forward the two words: efficacy and elegance" (Rohmer, [1955] 1985 89). Traducción propia 
un vehículo para un cine más 'personal', como un camino hacia argumentos cada vez más complejos para alcanzar un lenguaje cinematográfico comparable al literario"15 (Corrigan, 1999: 52).

Por otra parte, la política de los autores ha sufrido críticas derivadas de los excesos cometidos al desatender y rechazar ciertas obras que no son creadas por los considerados autores y, asimismo, defender aquellas firmadas por los nombres reconocidos, sin valorar objetivamente sus resultados (Santos-Fontenla, 1974). André Bazin, co-fundador de los Cahiers, fue el principal promotor de dicha idea, explicada en su artículo De la Politique des Auteurs ([1957] 2003), donde expresa que "deben poder existir, y efectivamente existen, esplendorosos estallidos en la producción por lo demás mediocre de un autor" (Bazin, [1957] 2003: 97). Bazin queda "distanciado del furor autoral del que sus pupilos hacen gala en sus textos" (Galindo-Pérez, 2015: 54) y propone asignar mayor atención a otros elementos como el texto, el contexto y la temática de la obra:

Al menos en cierta medida, el autor es siempre, para él mismo, su propio tema. Sea cual sea el guion, es la misma historia la que nos cuenta, o, por cuanto la palabra "historia" puede prestarse a confusión, digamos que es siempre la misma mirada y el mismo juicio moral vertidos sobre la acción y los personajes (Bazin, [1957] 2003: 101).

Desde otra perspectiva, se revela esencial en este análisis atender a la aplicación de la política de los autores en los círculos norteamericanos, que tuvo lugar en los años 60 gracias al crítico de la revista The Village Voice, Andrew Sarris ([1962] 1974). El autor cambió el término de "politique" por el de "theory" con la intención de evitar controversias (Cuevas, 1994; Pellejero, 2012), teniendo que aclarar posteriormente que el paradigma hace referencia a una actitud, entendida como un conjunto de valores (Sarris, 1968). Asimismo, apoya el concepto renovado del director de cine como artista, y apunta que, aunque la mayoría de ellos se ven supeditados al sistema de estudios, en realidad no tienen rivales en el proceso creador (Sarris 1967).

En Notes on the Auteur Theory in 1962 dicho crítico expone tres criterios para aceptar al director como autor:

La primera premisa de la teoría del autor es la competencia técnica de un director como criterio de valor. [...] La segunda premisa de la teoría del autor es la personalidad distinguible de un director como criterio de valor. Sobre un grupo de películas, un director debe exponer ciertas características de estilo recurrentes, que sirven como su firma. [...] La tercera y última premisa de la teoría del autor se refiere al significado interno, y al esplendor definitivo del cine como un arte. El significado interno es extrapolado desde la tensión entre la personalidad del director y su material ${ }^{16}$ (Sarris, [1962] 1974: 562).

Posteriormente, el británico Peter Wollen, en el capítulo The Auteur Theory-Signs and Meaning in the Cinema- (1969), aplica el estructuralismo y la teoría semiótica a la identidad del director. El crítico defiende su estudio basado en la relación entre la singularidad y la universalidad, es decir, en observar las características generales de la obra de un autor y, a su vez, las variantes en los temas y motivos de cada película (Stam, 2001; Pellejero, 2012; Galindo-Pérez, 2015). Desde dicho punto

15 Texto original: "both as a vehicle for a more 'personal' cinema and as pathway to more and more complex arguments for a language of the cinema comparable to a literary language" (Corrigan, 1999: 52). Traducción propia.

16 Texto original: "The first premise of the auteur theory is the technical competence of a director as a criterion of value. [...] The second premise of the auteur theory is the distinguishable personality of the director as a criterion of value. Over a group of films, a director must exhibit certain recurrent characteristics of style, which serve as his signature. [...] The third and ultimate premise of the auteur theory is concerned with interior meaning, and ultimate glory of the cinema as an art. Interior meaning is extrapolated from the tension between a director's personality and his material" (Sarris, [1962] 1974: 562). Traducción propia. 
de vista, Wollen (1969) analiza la trayectoria de directores como Howard Hawks o John Ford, afirmando que "lo importante a destacar, sin embargo, es que solo el análisis de la totalidad de su cine permite el momento de síntesis, cuando la crítica regresa a la película individual" ${ }^{17}$ (Wollen, 1969: 104). De este modo se pretendía "reforzar la validez del enfoque autorista, dándole una fundamentación más objetiva, más científica, que lograra erradicar los subjetivismos que muchas veces lastraban los intentos de los anteriores críticos de autor" (Cuevas, 1994: 157).

Finalmente, resulta necesario considerar algunas cuestiones sobre la autoría en el medio literario a través de las aportaciones de Roland Barthes ([1968] 1987) y Michel Foucault ([1969] 1999), cuyas nociones serán posteriormente aplicadas al campo cinematográfico (Galindo-Pérez, 2015).

Roland Barthes presenta su crítica en La mort de l'auteur ([1968] 1987), incidiendo en que la escritura destruye la identidad del que escribe, esto es, del potencial autor. Barthes señala la consideración de la autoría como signo de prestigio, y reconoce que la explicación de la obra es siempre buscada en quien la produjo: "La imagen de la literatura que es posible encontrar en la cultura común tiene su centro, tiránicamente, en el autor, su persona, su historia, sus gustos, sus pasiones” (Barthes, [1968] 1987: 66).

Michel Foucault expresa que lo importante no es estudiar la ausencia del autor, sino conocer lo que ello supone a través de su función y relación con el texto. En la conferencia titulada Qu'est -ce qu'un auteur? Foucault ([1969] 1999: 329-360) asigna la autoría a la persona que dice o escribe el discurso. Sin embargo, advierte la existencia de complejas intervenciones para alcanzar dicha atribución y, de este modo, anota cuatro funciones del autor en relación con el texto:

1. La "función- autor" conlleva la apropiación de un texto, responder ante lo escrito y sus consecuencias.

2. La "función-autor" varía según el discurso y el momento histórico, contribuyendo a la credibilidad del texto.

3. La "función-autor" supone valorar una serie de rasgos presentes en el texto, signos que remiten al autor.

4. La "función-autor" conlleva la existencia de una pluralidad de egos en el discurso, lo que Foucault denomina "parlante ficticio".

\subsection{La autoría en el medio televisivo}

Los trabajos citados permiten afirmar que la teoría del autor reivindicaba el poder y derechos del director de cine en detrimento del productor ejecutivo. Sin embargo, otros textos demuestran que en la "Época de los Estudios de Hollywood" existieron productores cuya intervención creativa sobre las películas fue determinante para el resultado final de las mismas (Kubey, 2009). En esta línea, Benshoff (2016: 66) expresa que "el productor era el hombre más importante de la producción, supervisando todos los aspectos de un proyecto e informando a los jefes del estudio"18.

Profesionales como David O. Selznick ejemplifican el perfil del productor ejecutivo-creativo, llegando a ser considerado co-autor de su obra cinematográfica (Pardo, 2000; Stam, 2001; Romaguera y Alsina, 2010; Benshoff, 2016). Selznick afirma-

17 Texto original: "the important thing to stress, however, is that it is only the analysis of the whole corpus which permits the moment of synthesis when the critic returns to the individual film" (Wollen, 1969: 104). Traducción propia.

18 Texto original: "it was the producer who was the most important man on the production, overseeing all aspects of a project and reporting to the studio bosses” (Benshoff, 2016: 66). Traducción propia. 
ba en una Conferencia en la Universidad de Columbia (1937) -posteriormente publicada en el libro MEMO from David O. Selznick (Behlmer, 1972)- que el productor debe ser capaz de reescribir o remontar una escena si es necesario, así como de indicarle al director cómo rodarla, sin limitarse a criticar la actuación del resto de profesionales. En este sentido, Selznick tenía "una profunda conciencia del carácter creativo de su profesión" (Pardo, 2009: 48).

Siguiendo esta premisa, la consideración del productor ejecutivo como autor alcanza su máxima expresión en la industria de la televisión, concretamente en la producción de series de ficción. Por lo tanto "la teoría del autor [...] tuvo que modificarse en su aplicación televisiva” (Stam, 2001: 113). De forma genérica, los académicos coinciden en asignar dicha autoría a productores ejecutivos -Steven Bochco, J.J. Abrams o Joss Whedon- que responden ante la máxima responsabilidad sobre la producción de series de televisión, mediante la supervisión de las diferentes fases del proceso (Newcomb y Alley, 1983; Caldwell, 1995; Lotz, 2009; Mann, 2009; Mittell, 2015; Perren y Schatz, 2015; Dunleavy, 2018). En este sentido, Kubey afirma:

En televisión el productor es la fuerza creativa clave: el productor crea y dirige el programa; y es, más que nadie, con rara excepción, la mente creativa y el gerente detrás de una serie y sus programas ${ }^{19}$ (Kubey, 2009: 18-19).

Por su parte, en el contexto histórico español, también existen productores de ficción televisiva -Jaime de Armiñán, Narciso Ibáñez Serrador, Adolfo Marsillach o Antonio Mercero- cuyo distintivo trabajo de guion, producción y dirección, supone su consideración como autores de la televisión española (García de Castro, 2002; Diego, 2010). En la última década podemos destacar, entre otros, a Javier Olivares, creador de El Ministerio del Tiempo (Cascajosa, 2015).

Resulta esencial apuntar que los estudios televisivos asocian la autoría a un productor ejecutivo-creativo (Mann, 2009; Mittell, 2015) que, en la era contemporánea, recibe el título no oficial de "showrunner" (Schatz, 2014). Kompare (2011: 98) define a dichos profesionales como "guionistas-productores que supervisaron y gestionaron la producción de la forma estética principal del medio: la serie en horario de máxima audiencia” ${ }^{20}$. Siguiendo esta línea, Steiner (2015: 183) expresa que "hoy en día la autoría en televisión está a menudo intrínsecamente ligada al showrunner, una posición en el nivel superior de la jerarquía de la producción de un drama televisivo, que también es identificado de manera variable como 'showrunner-auteur', 'writer-producer', o ‘hyphenate' de una serie de televisión”"21. De modo que, a pesar del carácter colaborativo del proceso:

Cuando se utiliza el término autor en televisión, generalmente se usa para describir al guionista-productor-creador de una serie dada, más que a los diversos guionistas y directores que podrían trabajar en la serie a lo largo de varios años ${ }^{22}$ (Benshoff, 2016: 63-64).

Sin embargo, no es exacto utilizar el término de "showrunner" como sinónimo de autor en televisión, pues dicha analogía no siempre se produce (Blakey, 2017). Debemos considerar la creación original del proyecto para evaluar la autoría del mis-

19 Texto original: "In television, the producer is the key creative force- the producer creates and runs the show, and it is the producer who, more than anyone else, with rare exception, is the creative mind and manager behind a series and its programs" (Kubey, 2009: 18-19). Traducción propia.

20 Texto original: "writer-producers who oversaw and managed production of the medium's primary aesthetic form, the primetime serial" (Kompare, 2011: 98). Traducción propia.

21 Texto original: "nowadays, authorship in television is often intrinsically tied to the showrunner, a position on top of a television drama's production hierarchy that is also variably labelled 'showrunner-auteur', 'writer-producer', or 'hyphenate' of a TV show” (Steiner, 2015: 183). Traducción propia.

22 Texto original: "When the term auteur is invoked about television, it is usually used to describe the writer-producer-creator of a given series, more so than the various writers and directors who might work on the show over the course of several years” (Benshoff, 2016: 63-64). Traducción propia. 
mo, ya que "solo en ciertas formas de producción televisiva observamos la elevación del showrunner al estatus de autor"23 (Newman y Levine, 2012: 39). Siguiendo esta idea, Gray y Johnson (2013) indican que el autor suele ser presentado como la persona que crea y transmite el producto, y recibe las críticas positivas y negativas sobre el mismo. Por lo tanto, "existen puntos de vista muy diferentes sobre la autoría, en función de los distintos modos de producción implicados” ${ }^{24}$ (Schatz, 2014: 42).

Por otra parte, como se ha expresado anteriormente, la teoría del autor asigna al director la competencia para aplicar una marca personal sobre sus películas y, así, configurar un estilo propio a lo largo de su filmografía (Cuevas, 1994). Sin embargo, en el medio televisivo la creatividad del director queda reducida: "Los directores de televisión tienen pocas oportunidades para poner su sello en un programa individual. De hecho, se les anima a no hacerlo, pues su capítulo no debe destacar como diferente del trabajo en serie ya establecido" 25 (Kubey, 2009: 225). En este caso, dicha responsabilidad es asignada al showrunner, que será el encargado de establecer un patrón a nivel narrativo, estético y temático; y mantener la consistencia e integridad de dicha visión con la evolución de la serie (Hadas, 2014; Benshoff, 2016; Dunne, 2017; Dunleavy, 2018). Cantor (1988: 111) se refiere a esta labor con el término de "quality control": "No solo se trata necesariamente de los aspectos artísticos, sino que también incluye hacer que la serie sea coherente manteniendo las historias dentro del concepto general de la misma”26. Por su parte, Campbell y Reeves (1990: 9) sugieren que la impronta de este productor ejecutivo está presente en tres partes del programa: "la orientación cultural, la estética cinematográfica, y la filosofía de la escritura" ${ }^{27}$. Las cuestiones mencionadas responden a un estilo personal y autobiográfico que podrá hacerse patente a lo largo de la trayectoria del showrunner en la producción de diferentes proyectos televisivos (Newman y Levine, 2012; Blakey, 2017).

Asimismo, debemos destacar la intervención del citado profesional en el departamento de guion (Schatz, 2014), ya que "la unión del guionista y el productor es clave en la producción de una serie dramática” ${ }^{28}$ (Dunne, 2007: 99). El showrunner conoce a la perfección la labor de este equipo, de donde suele proceder (Cantor, 1988; Cantor y Cantor, 1992; García-Fanlo, 2016; Fisk y Szalay, 2017). De manera que, de forma genérica, se puede afirmar que "el mayor impulso autoral recae en el guionista que idea la serie; él es quien, habitualmente, mantiene el control creativo ejerciendo como productor ejecutivo" (García-Martínez, 2014: 23). En este sentido, el proceso de escritura es considerado esencial para la visión creativa del proyecto (Mittell, 2015; Dunleavy, 2018) y, a través de la labor desarrollada por este writer-producer, hallamos conexiones entre la autoría en la literatura y el audiovisual (Corrigan, 1999; Fisk y Szalay, 2017).

Sin embargo, la actuación en el departamento de guion no es la única que debe ejercer el productor ejecutivo-creativo. A modo de síntesis, dicho profesional ejerce tres funciones fundamentales: establecer las líneas narrativas y supervisar su evolución, mantener el contacto con la cadena, y aplicar un modelo de producción acorde con las condiciones económicas

23 Texto original: "only in certain forms of television production do we observe the elevation of showrunner to the status of auteur" (Newman and Levine, 2012: 39). Traducción propia.

24 Texto original: "very different views of authorship are a function of the distinct modes of production involved" (Schatz, 2014: 42). Traducción propia.

25 Texto original: "Television directors have little opportunity to put their stamp on an individual program. Indeed, they are encouraged not to put a stamp on a program; it ought not to stand out as different from the series work already established" (Kubey, 2009: 225). Traducción propia.

26 Texto original: "Quality control does not necessarily refer just to artistic aspects of the film, but also includes making the series consistent by keeping the stories within the general series concept" (Cantor, 1988: 111). Traducción propia.

27 Texto original: "the cultural orientation; the cinematic look; and the writing philosophy" (Campbell and Reeves, 1990: 9). Traducción propia.

28 Texto original: "the marriage of writer and producer is key in producing the one-hour drama" (Dunne, 2007: 99). Traducción propia. 
de la empresa (Cantor, 1988). Por lo tanto, aunque la consideración del showrunner como autor televisivo se halla estrechamente asociada al control que ejerce en la parte creativa de la producción -guion, casting y edición- (Cantor y Cantor, 1992), se presenta imprescindible apuntar su responsabilidad sobre las cuestiones relacionadas con la gestión económica del proyecto de televisión (Mittell 2015). Ambas facetas son precisadas por Gray y Johnson al afirmar que:

El autor es un nodo a través del cual deben viajar los discursos de belleza, verdad, significado y valor; mientras que también es un nodo a través del cual deben viajar el dinero, el poder, el trabajo y el control de la cultura; y, al mismo tiempo, frecuentemente sirve como una figura que media permanentemente entre las grandes organizaciones y la audiencia ${ }^{29}$ (Gray y Johnson, 2013: 4).

Finalmente, resulta esencial anotar las consecuencias de la autoría televisiva sobre la promoción y difusión de la serie de ficción (Blakey, 2017; Jensen, 2017). Mittell (2015: 98) afirma que "la autoría frecuentemente funciona como una marca de distinción" ${ }^{30}$, y Steiner (2015: 183) añade que "el showrunner-auteur actúa como un garantizador de arte"31. En este sentido, Caldwell (1995: 14) expresa que "la emergencia del mito de la calidad en la televisión de los años 80 se debió, en parte, a que ésta ya no era simplemente anónima, como muchos teóricos habían sugerido"32. Newman y Levine (2012) estudian dicha cuestión como una herramienta para legitimar el medio televisivo desde una perspectiva cultural. Por su parte, Gray y Johnson (2013: 6) exponen: “La autoría [...] no es solo una cuestión de arte y expresión individual, sino también resultado de las estructuras sociales e institucionales que gobiernan la producción cultural, permitiendo, imponiendo y autorizando algunas formas, y limitando otras"33.

La firma autoral de los productores ejecutivos contribuye a la creación de una reputación que se emplea en la estrategia de marketing de sus futuros proyectos. Su nombre -“from the creator of"- es utilizado como reclamo publicitario para crear una serie de expectativas en la audiencia (Newman y Levine, 2012; Mittell, 2015; Jensen, 2017), o para unificar productos de diferente índole pertenecientes a un mismo universo transmedia (Hadas, 2014). Se trata de aplicar un modelo de promoción basado en la calidad y distinción creada alrededor del autor de la serie de ficción y, así, competir con los contenidos producidos por otros canales (Newman y Levine, 2012).

Además, la visibilidad proporcionada a los showrunners -en entrevistas, convenciones o conferencias- como parte del proceso de difusión y publicidad de una serie, permite equiparar el medio televisivo al literario o al cinematográfico desde esta perspectiva promocional (Newman y Levine, 2012). Por lo tanto, la labor del showrunner no finaliza con la emisión del proyecto acabado, sino que deberá participar e interactuar en actividades de diferentes medios y redes sociales, así como supervisar la creación de contenido para programas de televisión, páginas webs o secciones extra de DVD de series de televisión (Mann, 2009; Hills, 2013; Mittell, 2015; Perren y Schatz, 2015; Steiner, 2015).

29 Texto original: "The author is a node through which discourses of beauty, truth, meaning, and value must travel, while also being a node through which money, power, labour, and the control of culture must travel, and while frequently serving as the mediating figure standing between large organizations and the audience" (Gray and Johnson, 2013: 4). Traducción propia.

30 Texto original: "authorship frequently functions as a marker of distinction” (Mittell, 2015: 98). Traducción propia.

31 Texto original: "the showrunner-auteur acts as a perceived guarantor of art" (Steiner, 2015: 183). Traducción propia.

32 Texto original: "part of the emergence of the quality myth in 1980s television was that television was no longer simply anonymous as many theorists had suggested" (Caldwell, 1995: 14). Traducción propia.

33 Texto original: "authorship is [...] not just a question of art and individual expression, but also of social and institutional structures that govern cultural production, enabling, compelling, and authorizing some forms while constraining others” (Gray and Johnson, 2013: 6). Traducción propia. 
Revisión histórica y conceptual de la autoría y sus implicaciones en el medio televisivo...

\section{Conclusiones}

Los resultados de la investigación sobre la autoría en la producción televisiva a través de la revisión de la Politique des Auteurs francesa y su posterior aplicación a la televisión estadounidense, nos permiten extraer una serie de conclusiones al respecto. En este punto, enfatizamos la consideración del productor ejecutivo-creativo como autor de las series de ficción televisiva, así como su trabajo e implicaciones en el proceso de producción desde una perspectiva autoral.

En primer lugar, la asignación de la autoría se configura como una tarea compleja que ha sido estudiada en diferentes medios -literatura, cine, televisión- hallando importantes influencias entre ellos. Asimismo, dichas cuestiones se ven notablemente condicionadas por un contexto cultural y social determinado, y deben entenderse dentro del sistema colaborativo de la producción audiovisual, sin que ello impida la existencia de un autor individual.

Las críticas a la autoría en la industria televisiva se dirigen al hecho de hallarnos ante el resultado de un trabajo conjunto donde participan diferentes profesionales de la empresa productora, junto con las cuestiones técnicas y económicas, y la intervención de la cadena de televisión y la audiencia, lo que dificulta la consideración de un autor individual. Sin embargo, las teorías sobre el cine y la televisión revelan que dicha idea no tiene por qué suponer la inexistencia de una persona encargada de guiar la actuación de los demás trabajadores, pudiendo adquirir la autoría del proyecto. Así, este perfil ejerce la máxima responsabilidad sobre la creación y el desarrollo del mismo, aplicando una visión creativa personal, y asegurando su consistencia a lo largo de la producción. En el cine dicho crédito suele ser desarrollado por el director de la película; sin embargo, en la industria televisiva es más frecuente asignar la autoría al productor ejecutivo-creativo de las series de ficción, designado con el término de “showrunner" en la era contemporánea.

En ambos medios, los estudios destacan la importancia de la huella personal que el autor aplica sobre la obra audiovisual, la cual suele ser reiterativa durante su trayectoria laboral y, al ser reconocida por la audiencia, permite crear una serie de expectativas ante futuros proyectos. Es imprescindible que dicha visión no sea desvirtuada por los miembros de cada departamento, lo que resulta especialmente importante en las series de ficción, compuestas por varios capítulos escritos y dirigidos por diferentes profesionales. Por lo tanto, el showrunner, siendo o no creador de la idea original, deberá preservar la integridad y continuidad de la misma, adaptando las diferentes aportaciones a dicho concepto, lo que repercute positivamente en la calidad del producto emitido.

Por otra parte, la popularidad que han adquirido las series de ficción en el siglo XXI ha propiciado la legitimización del medio televisivo desde una perspectiva cultural, gracias a la existencia de dicha autoría desarrollada por este productor ejecutivo. La actuación del showrunner sobre las estrategias de promoción y marketing del proyecto acabado, las relaciones establecidas con la audiencia gracias a las redes sociales y a las opciones transmedia, y la intervención de la cadena de televisión en la creación de una imagen de marca, influyen de forma patente en la configuración social y cultural de las series de ficción televisiva como un producto de calidad.

A partir de los datos estudiados, tomando como referentes la autoría en la literatura y en el cine, se valora la consideración del showrunner como autor si atendemos a la supervisión que realiza sobre el proceso de producción de las series de televisión en su totalidad. Concretamente, destacamos la labor desarrollada en el departamento de guion, lo que lo asemejaría al autor literario; así como el control que ejerce durante el rodaje y la postproducción, acercándolo al liderazgo que el 
director desempeña en el medio cinematográfico. En este punto, junto con el estudio de las funciones y competencias del productor ejecutivo-creativo durante el proceso de producción, atendemos a la posibilidad de evaluar la autoría a través del análisis de las series de ficción televisiva y su marca autoral narrativa, estética y temática, motivando el interés de profundizar en esta investigación con futuras aplicaciones.

\section{Referencias bibliográficas}

Astruc, A. [1948] (1999): “The Birth of a New Avant-Garde: 'La caméra-stylo”, en Corrigan, T. (ed.): Film and Literature: An Introduction and Reader. Upper Saddle River, NJ: Prentice-Hall, pp. 158-162.

Baecque, A. (2013): La política de los autores: Manifiestos de una generación de cinéfilos. Barcelona: Paidós.

Barthes, R. [1968] (1987): “La muerte del autor”, en El susurro del lenguaje. Más allá de la palabra y de la escritura. Barcelona: Paidós, pp. 65-71.

Bazin, A. [1957] (2003): "De la política de los autores", en Baecque, A. (ed.): La Política de los autores: Manifiestos de una generación de cinéfilos. Barcelona: Paidós, pp. 91-105.

Behlmer, R. (1972). MEMO from David O. Selznick. New York: The Viking Press.

Bennett, T. (2014): Showrunners: The Art of Running a TV Show. London: Titan Books.

Benshoff, H. M. (2016): Film and television analysis. New York: Routledge, Taylor \& Francis Group.

Blakey, E. (2017): "Showrunner as Auteur: Bridging the Culture/Economy Binary in Digital Hollywood", Open Cultural Studies, vol. 1, n. 1, pp. 321-332. Recuperado de https://doi.org/10.1515/culture-2017-0029 [Consultado el 10/01/2018].

Caldwell, J. T. (1995): Televisuality. Style, Crisis, and Authority in American Television. New Brunswick: Rutgers University Press.

Campbell, R.; y Reeves, J. L. (1990): “Television Authors: The Case of Hugh Wilson”, en Thompson, R.J.; y Burns, G. (eds.): Making Television: Authorship and the Production Process. New York: Praeger, pp. 3-18.

Cantor, M. G. (1988): The Hollywood TV producer. His work and his audience ( $2^{\text {nd }}$ ed.). New Brunswick and Oxford: Transaction Books.

Cantor, M. G.; y Cantor, J. M. (1992): Prime-time television. Content and control ( $2^{\text {nd }}$ ed.). Newbury Park: The Sage Commtext Series.

Cascajosa, C. (2015): Dentro de El Ministerio del Tiempo. Madrid: Léeme Libros.

Casetti, F. (1994): Teorías del cine, 1945-1990. Madrid: Cátedra.

Chaudhuri, A. (2013): "Auteur Theory and its Implications", International Journal of Advancements in Research \& Technology, vol. 2, n. 11, pp. 77-89. Recuperado de http://www.ijoart.org/docs/Auteur-Theory-and-its-implications.pdf [Consultado el 20/04/2018].

Corrigan, T. (ed.) (1999): Film and Literature: An Introduction and Reader. Upper Saddle River, NJ: Prentice-Hall. 
Revisión histórica y conceptual de la autoría y sus implicaciones en el medio televisivo...

Cuevas, E. (1994): “Notas sobre la 'teoría del autor' en ficciones audiovisuales”, Communication \& Society, vol. 7, n. 1, pp. 155-164. Recuperado de https://www.unav.es/fcom/communication-society/es/articulo.php?art_id=224\#C02 [Consultado el 13/10/2018].

Diego, P. (2010): La ficción en la pequeña pantalla. Navarra: EUNSA.

Dunleavy, T. (2018): Complex Serial Drama and Multiplatform Television. New York: Routledge.

Dunne, P. (2007): “Inside American Television Drama. Quality is Not What is Produced, But What it Produces", en McCabe, J.; y Akass, K. (eds.): Quality TV: Contemporary American Television and Beyond. New York: I.B. Tauris, pp. 98-110.

Fisk, C.; y Szalay, M. (2017): "Story Work: Non-Proprietary Autonomy and Contemporary Television Writing”, Television \& New Media, vol. 18, n. 7, pp. 605-620. Recuperado de https://doi.org/10.1177/1527476416652693 [Consultado el $13 / 10 / 2018]$.

Foucault, M. [1969] (1999): “¿Qué es un autor?”, en Entre filosofía y literatura. Obras esenciales. Vol. I. Barcelona: Paidós, pp: 329-360.

Galindo-Pérez, J. M. (2015): “Del autor en la historia del cine. Revisiones y nuevas vías”, Zer - Revista De Estudios De Comunicación, vol. 20, n. 39, pp. 49-66. Recuperado de https://doi.org/10.1387/zer.15517 [Consultado el 13/10/2018].

García de Castro, M. (2002): La ficción televisiva popular. Una evolución de las series de televisión en España. Barcelona: Gedisa.

García-Fanlo, L. (2016): El lenguaje de las series de televisión. Buenos Aires: Editorial Universitaria de Buenos Aires (EUDEBA). García-Martínez, A. N. (2014): “El fenómeno de la serialidad en la tercera edad de oro de la televisón”, en Fuster, E. (ed.): $L a$ figura del padre nella serialitá televisiva. Roma: Pontificia Universitá della Santa Croce, pp. 19-42.

Gray, J.; y Johnson, D. (eds.) (2013): Companion to Media Authorship. Massachusetts: Wiley-Blackwell.

Guerrero, E. (2013): Guión y producción de programas de entretenimiento. Navarra: EUNSA.

Hadas, L. (2014): "Authorship and Authenticity in the Transmedia Brand: The Case of Marvel's Agents of S.H.I.E.L.D.", Networking Knowledge: Journal of the MeCCSA Postgraduate Network, vol. 7, n. 1, pp. 7-17. Recuperado de https:/ ojs.meccsa. org.uk/index.php/netknow/article/view/332 [Consultado el 14/10/2018].

Hills, M. (2013): "From Chris Chibnall to Fox: Torchwood's Marginalized Authors and Counter-Discourses of TV Authorship", en Gray, J., y Johnson, D. (eds.): Companion to Media Authorship. Massachusetts: Wiley-Blackwell, pp. 200-220.

Jensen, M. (2017): “From the Mind of David Simon: A Case for the Showrunner Approach", International Journal of TV Serial Narratives, vol. 3, n. 2, pp. 31-42. Recuperado de https://doi.org/10.6092/issn.2421-454X/7610 [Consultado el 13/10/2018]. Johnson, D. (2013): “Participation is Magic: Collaboration, Authorial Legitimacy, and the Audience Function”, en Gray, J., y Johnson, D. (eds.): Companion to Media Authorship. Massachusetts: Wiley-Blackwell, pp. 133-157.

Kirkpatrick, S. (2015): Writing for the Green Light. How to Make your Script the One Hollywood Notices. New York: Focal Press. Kompare, D. (2011): “More 'Moment of Television': Online Cult Television Authorship”, en Kackman, M. et al. (eds.): Flow TV: Television in the Age of Media Convergence. New York: Routledge, pp. 95-113. 
Kubey, R. (ed.) (2009): Creating Television. Conversations with the People Behind 50 Years of American TV. New York: Routledge.

Lotz, A. (2009): “Industry-Level Studies and the Contributions of Gitlin's Inside Prime Time”, en Mayer, V. et al. (eds.): Production Studies. Cultural Studies of Media Industries. New York: Routledge, pp. 25-38.

Maio, B. (2011): “HBO e la politica del network-autore”, en Pérez-Gómez, M. A. (ed.): Previously On. Interdisciplinary Studies on TV Series in the Third Golden Age of Television. Sevilla, España: Biblioteca de la Facultad de Comunicación de la Universidad de Sevilla, pp. 279-289.

Mann, D. (2009): “It's Not TV, It’s Brand Management TV: The Collective Author(s) of the Lost Franchise”, en Mayer, V. et al. (eds.): Production Studies. Cultural Studies of Media Industries. New York: Routledge, pp. 99-114.

Mittell, J. (2015): Complex TV: The poetics of contemporary television storytelling. New York: New York University Press.

Newcomb, H.; y Alley, R.S. (1983): The Producer's Medium. Conversations with Creators of American TV. Oxford: Oxford University Press.

Newman, M. Z.; y Levine, E. (2012): Legitimating Television: Media Convergence and Cultural Status. Nueva York: Routledge. Pardo, A. (2000): "Creativity in Film Production: The Film Producer as a Creative Force", Communication \& Society, vol. 13, n. 2, pp. 227-249. Recuperado de http://www.unav.es/fcom/communication-society/es/resumen.php?art_id=128 [Consultado el 17/04/2019].

_ (2009): “El productor creativo: ¿tautología o excepción?”, en Marzal Felici, J.; y Gómez Tarín, F.J. (eds.): El productor y la producción en la industria cinematográfica. Madrid: Complutense, pp. 45-66.

_ (2014): Producción Ejecutiva de Proyectos Cinematográficos (2a ed.). Navarra: EUNSA.

Pellejero, E. (2012): “Author's Politics and Death of Man: Notes for a Genealogy of Film Criticism", Sesión no numerada: Revista de Letras y Ficción Audiovisual, n. 2, pp. 29-53. Recuperado de https://dialnet.unirioja.es/servlet/articulo?codigo=3877156 [Consultado el 23/09/2018].

Perren, A. (2011): "In Conversation: Creativity in the Contemporary Cable Industry", Cinema Journal, vol. 50, n. 2, pp. 132138. Recuperado de http://www.jstor.org/stable/41240699 [Consultado el 03/10/2018].

Perren, A.; y Schatz, T. (2015): “Theorizing television’s Writer-Producer: Re-viewing The Producer's Medium”, Television \& New Media, vol. 16, n. 1, pp. 86-93. Recuperado de https://doi.org/10.1177/1527476414552907 [Consultado el 03/10/2018]. Romaguera, J.; y Alsina, H., (eds.) (2010): Textos y manifiestos del cine. Estética. Escuelas. Movimientos. Disciplinas. Innovaciones ( $5^{\mathrm{a}}$ ed.). Madrid: Cátedra.

Rohmer, E. [1955] (1985): “Rediscovering America”, en Hillier, J. (ed.): Cahiers du Cinéma. The 1950s: Neo-Realism, Hollywood, New Wave. Cambridge: Harvard University Press, pp. 88- 93.

Sáinz-Sánchez, M. (1999): El Productor Audiovisual. Madrid: Síntesis.

Sanderson, J. D. (2005): ¿Cine de autor? revisión del concepto de autoría cinematográfica. Murcia:Vicerrectorado de Extensión Universitaria.

Santos-Fontenla, C. (ed.) (1974): La política de los autores. Madrid: Editorial Ayuso. 
Revisión histórica y conceptual de la autoría y sus implicaciones en el medio televisivo...

Sarris, A. [1962] (1974): “Notes on the auteur theory in 1962", en Mast, G.; Cohen, M.; y Braudy, L. (eds.): Film Theory and Criticism: Introductory Readings. Nueva York: Oxford University Press, pp. 561-564.

_ (1967): Interviews with Film Directors. New York: Bobbs-Merrill.

_ (1968): The American Cinema: Directors and Directions. New York: E.P. Dutton \& Co. Inc.

Schatz, T. (2014): “Film Studies, Cultural Studies, and Media Industries Studies", Media Industries Journal, vol. 1, n. 1, pp. 39-43. Recuperado de http://dx.doi.org/10.3998/mij.15031809.0001.108 [Consultado el 25/09/2018].

Scott, J. F. (1975): Film: The Medium and the Market. New York: Holt, Rinehart and Winston.

Stam, R. (2001): Teorías del cine: Una introducción. Barcelona: Paidós.

Steiner, T. (2015): "Steering the Author Discourse: The Construction of Authorship in Quality TV, and the Case of Game of Thrones", International Journal of TV Serial Narratives, vol. 1, n. 2, pp. 181-192. Recuperado de https://doi.org/10.6092/ issn.2421-454X/5903 [Consultado el 10/09/2018].

Thompson, R. J.; y Burns, G. (eds.) (1990): Making Television: Authorship and the Production Process. New York: Praeger.

Tous-Rovirosa, A. (2009). "El concepto de autor en las series norteamericanas de calidad", en Serafim, J. F. (ed.): Autor e autoria no cinema e na televisão. Salvador: EDUFBA, pp. 121-169.

Wollen, P. (1969): “The Auteur Theory”, en Signs and Meaning in the Cinema. Bloomington: Indiana University Press, pp. 74-115. 